\title{
Thermomechanical Analysis of Vibrations of Silent Block
}

\author{
V. Szüle ${ }^{1}$, B. Pere ${ }^{2}$ \\ ${ }^{1}$ University of Széchenyi István, Department of Applied Mechanics \\ Square Egyetem 1, 9026 Győr, Hungary \\ Phone: +36 96503400 \\ e-mail: szule.veronika@sze.hu \\ ${ }^{2}$ University of Széchenyi István, Department of Applied Mechanics \\ Square Egyetem 1, 9026 Győr, Hungary \\ Phone: +36 96503400
}

\begin{abstract}
Vehicle components made of rubber or rubber like polymers usually exhibit large deformations. Cyclic deformations may induce increasing in temperature in elastic materials. In this paper after the summary of the basic physical laws and the description of basement of continuummechanics, taking into account the Neo-Hooke material law, an example will be presented which allows to calculate strain- and temperature changes.
\end{abstract}

Keywords: rubber, rubber material laws, high deformations, thermodynamics, Neo-Hooke material law

\section{Introduction}

Generally, the most frequently used structural materials are metals which have high strength and stiffness. However, there are many cases, when other important properties come to the fore as well as high deformation by elastic behavior, high viscosity namely good damping effect. The metals do not have these above mentioned properties, but the rubber does. The rubber- thanks to its elastic behavior is able to establish an elastic connection between hard and brittle structural elements, however, has high load carrying capacity.

Rubber can be classified as a so-called hyperelastic polymer which has a typical geometrical and material non-linear behavior. It means that the relationship between 
displacements and internal forces can be described by functions whose order is higher than linear. The geometrical nonlinearity is easy to handle mathematically, however the material nonlinearity is only described approximately [1] [2]. Independent of the experimental investigations which deal with the material behavior of rubber, a number of theoretical works treated rubber as an ideally nonlinear elastic, in particular hyperelastic material. One of the properties of the constitutive equations of hyperelastic material is that stresses are derived from stored elastic energy function. Hyperelasticity is a particularly convenient constitutive equation given its simplicity and it constitutes the basis for more complex material models such as elastoplasticity, viscoplasticity, and viscoelasticity [1].

Ogden [3] [4] was able to obtain very good correlation with the experiments of Treloar. Above that, his strain energy function fulfills all necessary mathematical and physical requirements. Furthermore, a number of material laws for rubber can be found in literature [5] [6] for example the Neo-Hooke-, the Mooney-Rivlin, the Yeoh-, and the Arruda-Boyce material models. Their applicapability largely depends on the stress.

Thus, description of the behaviour of rubber and rubberlike materials it is quite difficult from several aspects. The nonlinear behaviour and the fact that the strain can be comparable to the original measure of the parts shows that the models used for small strains cannot be applied even besides compromise.

Furthermore, the task becomes more complicated because of some features of rubber parts. The temperature of rubber increases significantly. Therefore, the temperature- and displacement fields are coupled, and it means that special solving algorithms are required [7]. So the equations of mechanics and thermodynamics are coupled.

As described above, the goals of this paper are the following:

It is necessary to summarize the applied equations and the basic physical laws which are responsible for the theoretical background [8]. Clarification of these relationships is essential because the material laws of rubber cannot violate those basic physical laws. It is necessary to extend these relationships like equilibrium of linear momentum and equilibrium of angular momentum, the first and second law of thermodynamics to high deformation of rubber and rubberlike polymers. After it, it will follow the numerical solution and computer simulation of the thermomechanical problem by using of the Neo-Hooke material law. 


\section{Notation}

Table 1. Notation

\begin{tabular}{|l|l|}
\hline \multicolumn{2}{|l|}{ Mechanics: } \\
\hline$\vec{t}$ & Force per unit surface/traction, acting on the material body \\
\hline$\vec{f}$ & Force per unit volume, acting on the material body \\
\hline$\underline{\underline{\sigma}}$ & Cauchy-stress tensor \\
\hline$\vec{v}$ & Velocity of the material body \\
\hline$\rho$ & Mass density \\
\hline Continuum & mechanics \\
\hline$\underline{F}$ & Deformation gradient tensor \\
\hline$\vec{r}$ & Position vector \\
\hline$J$ & Volume ratio \\
\hline Thermodynamics \\
\hline$\varepsilon$ & Energy supply density \\
\hline$\vec{q}$ & Heat flow vector \\
\hline$h$ & Heat source \\
\hline$\eta$ & Entropy density \\
\hline$\varphi$ & Free energy \\
\hline$\varphi_{0}$ & Free energy at the reference temperature \\
\hline$\hat{c}$ & Specific heat \\
\hline Vector calculus \\
\hline$\vec{a} \cdot \vec{b}$ & Scalar product (dot product) of two vectors \\
\hline$\nabla$ & Vector product (cross product) of two vectors \\
\hline
\end{tabular}




\section{Basics of continuum mechanics}

Consider a fixed reference configuration of a body corresponding to a fixed reference time $t_{0}$. The position of a typical point may be identified by the position vector $\vec{R}$. The reference configuration is assumed to be stress-free and possesses a homogeneous reference temperature value $T_{0}(>0)$.

Furthermore the quantities of the $t_{0}$ moment will indicated by capital letters and the quantities of an optional $t$ moment will indicated by small letters. Thus, the position vector of material particle is $\vec{r}$ at time $t$. The position of the body is called current configuration at time $t$ where each quantity depends on time. During the reference configuration the quantities do not depend on time, because of the fixed time moment. A map of the reference configuration to a current configuration is characterized by the macroscopic motion $\vec{r}(t)=\vec{\chi}(\vec{R}, t)$ [8].

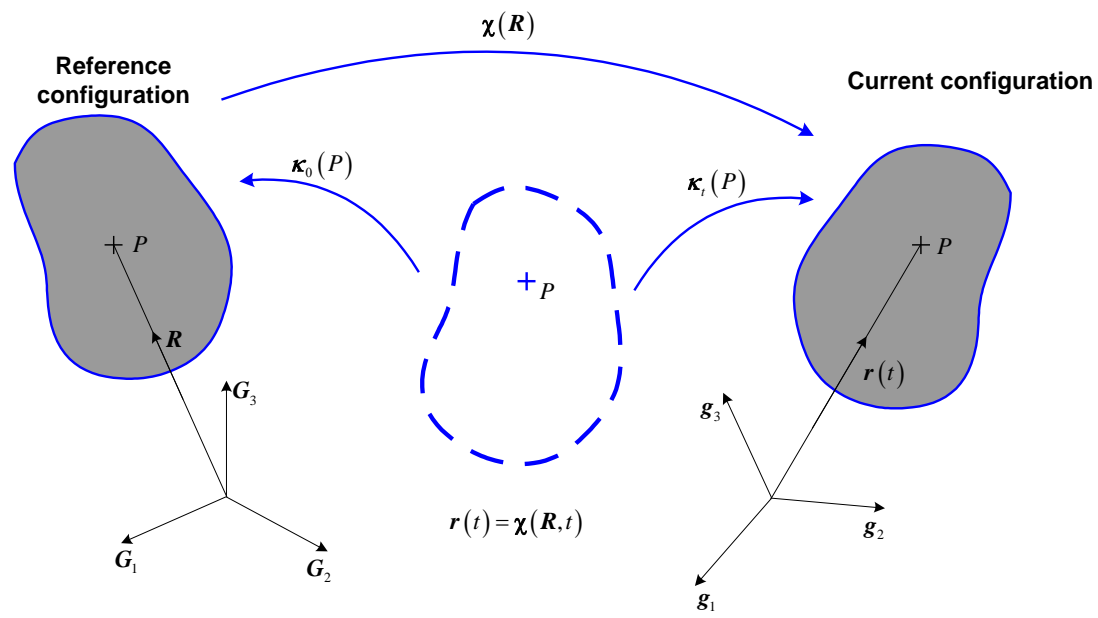

Figure 1. Connection between the reference and current configuration

Fig. 1 shows the connection between the reference and current configuration, i.e. movement and deformation of the body is determined by the $\vec{r}(t)=\vec{\chi}(\vec{R}, t)$ maping, so the $\mathrm{P}$ point which was originally determined by the $\vec{R}$ position vector moves to 
the place determined by the $\vec{r}$ position vector. As a measure of the thermoelastic deformation we use the deformation gradient $\underline{=}=\operatorname{grad} \vec{\chi}=\frac{\partial \vec{\chi}(\vec{R})}{\partial \vec{R}}=\vec{\chi}(\vec{R}) \circ \nabla_{0}$, where the zero index of the differential operator $\nabla_{0}$ means that the derivation has to be done in the reference configuration. Deformation gradient is the base variable of the continuum mechanical task and by using it the other quantities of the problem can be derived.

\section{Governing equations}

\subsection{Equlibrium of linear momentum}

Let us consider a hyperelastic continuum body in the current configuration. Its volume is indicated by $v$, and its surface is indicated by $a$. The integral formulation of equlibrium of linear momentum in the current configuration is:

$$
\frac{d}{d t} \int_{(v)} \vec{v} \rho d v=\int_{(a)} \vec{t} d a+\int_{(v)} \vec{f} d v
$$

where $\vec{v}$ is the velocity of one point of the continuum body, $\rho$ is the mass density of the material of the body, $\vec{t}$ force per unit surface/traction, $\vec{f}$ force per unit volume.

During the solution of the problem we need to know the differential formulation of the equlibrium of linear momentum, which can be generated by the Gauss's theorem:

$$
\rho \dot{\vec{v}}=\underline{\underline{\sigma}} \cdot \nabla+\vec{f}
$$

where $\vec{v}$ is the velocity of one point of the continuum body, $\rho$ is the mass density of the material of the body, $\vec{t}$ force per unit surface, $\vec{f}$ force per unit volume, $\underline{\underline{\sigma}}$ Cauchy-stress tensor, namely the $\vec{t}$ traction can be expressed by the Cauchy-stress as follows: $\vec{t}=\underline{\underline{\sigma}} \cdot \vec{n}$. 


\subsection{Equilibrium of angular momentum}

Let us consider a hyperelastic continuum body in the current configuration, its volume is indicated by $v$, and its surface is indicated by $a$. The integral formulation of equilibrium of angular momentum in the current configuration is:

$$
\frac{d}{d t} \int_{(v)} \vec{r} \times \vec{v} \rho d v=\int_{(a)} \vec{r} \times \vec{t} d a+\int_{(v)} \vec{r} \times \vec{f} d v
$$

Hereafter, we use the differential form of the equilibrium of angular momentum in the current configuration:

$$
\vec{r} \times(\rho \dot{\vec{v}}-\underline{\underline{\sigma}} \cdot \nabla-\vec{f})=\stackrel{\downarrow}{\vec{r}} \times \underline{\underline{\sigma}} \cdot \nabla
$$

The differential form of the balance of moments can be expressed by the following equality $\underline{\underline{\sigma}}=\underline{\underline{\sigma}}^{T}$. The consequence of this formula is that the stress tensor is symmetric.

\subsection{The first law of thermodynamics}

Cyclic finite deformations cause exoterm process in hyperelastic materials. The classical thermodynamical characterization of the continuum body is presented by Holzapfel and Simo [8], where the laws of thermodynamics, the Helmholtz free energy and the heat conduction play a significant role.

The form of the first law of thermodynamics in the current configuration is:

$$
\frac{d}{d t} \int_{(v)} \dot{\varepsilon} \rho d v=-\int_{(a)} \vec{q} \cdot \vec{n} d a+\int_{(v)} h d v+\underbrace{\int_{(a)} \vec{v} \cdot \vec{t} d a+\underbrace{\int_{v} \vec{v} \cdot \vec{f} d v}_{(v)}}_{\text {Performance of forces on surface Performance of forces on volume }}
$$

where $\varepsilon$ is the energy density (energy per unit mass), $\vec{q}$ is the heat flux, $h$ is the heat source.

Using Gauss's theorem the differential form of the Eq. (5) is: 


$$
\dot{\varepsilon} \rho=[-\nabla \cdot \vec{q}+h]+[\underline{\underline{\sigma}} \cdot \underline{=}+\rho \vec{v} \cdot \dot{\vec{v}}]
$$

where $\vec{v} \cdot \dot{\vec{v}}=\frac{d}{d t}\left(\frac{v^{2}}{2}\right), \frac{d}{d t}\left(\varepsilon-\frac{v^{2}}{2}\right) \rho=[-\nabla \cdot \vec{q}+h]+\underset{=}{\sigma} \cdot \stackrel{\underline{l}, l}{=}=\vec{v} \circ \nabla$ is the velocity gradient, $\underline{\underline{l}}=\underline{\underline{F}} \cdot \underline{F^{-1}}$. Introducing the internal energy per unit mass $e=\varepsilon-\frac{v^{2}}{2}$ the first law of thermodynamics in the current configuration has the following form:

$$
\dot{e} \rho=[-\nabla \cdot \vec{q}+h]+\underline{\underline{\sigma}} \cdot \stackrel{\cdot l}{=}
$$

\subsection{The second law of thermodynamics}

The behaviour of viscoelastic materials is described by the second law of thermodynamics. If the material is viscous and does stress-relaxing which is caused by stress, it can happen only in one direction. The stress decreases or increases. The status of an isolated system is proceeding to the thermal equilibrium.

The second law of thermodynamics can be expressed by the following inequality:

$$
\frac{d}{d t} \int_{(v)} \eta \rho d v \geq-\int_{(a)} \frac{\vec{q} \cdot d \vec{a}}{T}+\int_{(v)} \frac{h d v}{T}
$$

where $\eta$ is the entropy density, $T$ is the absolute temperature.

Using Gauss's theorem and the $\nabla \cdot\left(\frac{a}{b}\right)=\frac{\nabla \cdot a}{b}-\frac{a \cdot \nabla b}{b^{2}}$ identity the differential form of Eq. (8) is:

$$
\dot{\eta} T \rho \geq-\nabla \cdot q+\frac{q \cdot \nabla T}{T}+h
$$

It will be expedient to change the variable of the exercise from entropy to temperature by applying the Legendre-transformation and by using the Helmholtz free energy

$$
\psi=e-\eta T
$$

Substituting Eq. (10) into Eq. (7) and subtract Eq. (7) from Eq. (10) the following expression is generated: 


$$
-(\dot{\psi}+\eta \dot{T}) \rho+\underset{=}{\sigma} \cdot \stackrel{l}{=}-\frac{q \cdot \nabla T}{T} \geq 0
$$

this is the Clausius-Duhem inequality [6].

\section{Constitutive model}

\subsection{Stress state of elastic element}

The property of an elastic element is that the total mechanical energy is reversible. The free energy of the body is the function of the strain and temperature. Dissipation comes only from heat conduction.

\subsection{Structure of free energy function}

In order to make the further calculations easier it is necessary to split the Eq. (10) to temperature-dependent and temperature-independent parts [12]. Based on known functions $\tilde{\psi}_{0}(\underline{\underline{C}})$ and $e_{0}(\underline{\underline{C}})$ for the free energy and the internal energy at a given reference temperature $T_{0}$ and the given heat capacity $\hat{c}$ at a reference temperature, one obtains the following general structure for the thermoelastic free energy from the Eq. (10):

$$
\psi(\underline{\underline{C}}, T)=\tilde{\psi}(\underline{\underline{C}}, \tilde{T})=\frac{T}{T_{0}} \psi_{0}(\underline{\underline{C}})+\left(1-\frac{T}{T_{0}}\right) e_{0}(J)+\int_{T_{0}}^{T} \hat{c}(\underline{\underline{C}}, \tilde{T})\left(1-\frac{T}{\tilde{T}}\right) d \tilde{T},
$$

where $\underline{\underline{C}}$ is the right Cauchy-Green strain tensor [12].

\subsection{Neo-Hooke material law}

In the following section we are going to investigate the isotrop materials and we are going to apply the Neo-Hookean material law. It means that $\psi_{0}$ which is used in free energy depends on the scalar invariant of the right Cauchy-Green strain tensor. The internal energy is zero applying the entropic theory and the $c$ heat capacity is constant with good approximation. In the case of Neo-Hooke material the free energy is:

$$
\psi(\underline{\underline{C}}, T)=\tilde{\psi}(\underline{\underline{C}}, \tilde{T})=\frac{T}{T_{0}} \psi_{0}(\underline{\underline{C}})+\left(1-\frac{T}{T_{0}}\right) e_{0}(J)+\int_{T_{0}}^{T} \hat{c}(\underline{\underline{C}}, \tilde{T})\left(1-\frac{T}{\tilde{T}}\right) d \tilde{T}
$$

where 
$\mu$ is the shear modulus,

$C_{I}$ is the first scalar invariant of the right Cauchy-Green strain tensor, $C_{I}=\underline{\underline{C} \cdot \cdot I}$ [2].

\subsection{Equation of heat conduction}

Starting from the first law of thermodynamics and introducing the internal energy and changing the variable from entropy to temperature, the equation will have the next form:

$$
\rho_{0} c \dot{T}=\left(\underline{\underline{S}}-\rho_{0} \frac{\partial \psi}{\partial \underline{\underline{C}}}\right) \cdot \cdot \frac{1}{2} \underline{\underline{\dot{C}}}+\rho_{0} \frac{\partial^{2} \psi}{\partial T \partial \underline{\underline{C}}} \cdot \cdot \underline{\underline{C}} T-q_{0} \nabla_{0}+h_{0},
$$

where $\left(\underline{\underline{S}}-\rho_{0} \frac{\partial \psi}{\partial \underline{\underline{C}}}\right) \cdot \frac{1}{2} \underset{\underline{C}}{\dot{C}}$ is the non-recoverable part of the mechanical power, which is zero in the case of a pure elastic element [1], [2]. In this case the reological model is regarded to be a pure elastic element. So the free energy of the body is characterized by the deformation and temperature:

$$
\psi=\psi(\underline{\underline{C}}, T) \text { or }\left(\underline{\underline{S}}-\rho_{0} \frac{\partial \psi}{\partial \underline{\underline{C}}}\right) \cdot \cdot \frac{1}{2} \underline{\underline{C}}=0, \text { így } \underline{\underline{S}}=\rho_{0} \frac{\partial \psi}{\partial \underline{\underline{C}}}
$$

Furthermore, we are assuming that there are not heat sources in the rubber and the temperature field shows homogeneous distribution. $h_{0}=0, q_{0}=0$

Thus, the equation of the heat conduction is the following:

$$
c \dot{T}=T \frac{\partial^{2} \psi}{\partial T \partial \underline{\underline{C}}} \cdot \dot{\underline{C}}
$$

\section{Example}

Let us consider the mechanical model of a silent block, thus the A, B, C axisymmetric bodies (see Fig.2). The $\mathrm{A}$ and $\mathrm{C}$ bodies are rigid bodies, and $\mathrm{B}$ is a deformable one. Regarding the structure of the silent block it consists of two metal elements whose are connected by the rubber which is vulcanized between them. The 
inside rubber part provides a non-linear elastic connection between the two metal elements in the following way: it transfers loads however filters out the harmful vibrations, i.e. it has damping effect. All three bodies are axysymmetric and their symmetry axes are the same.

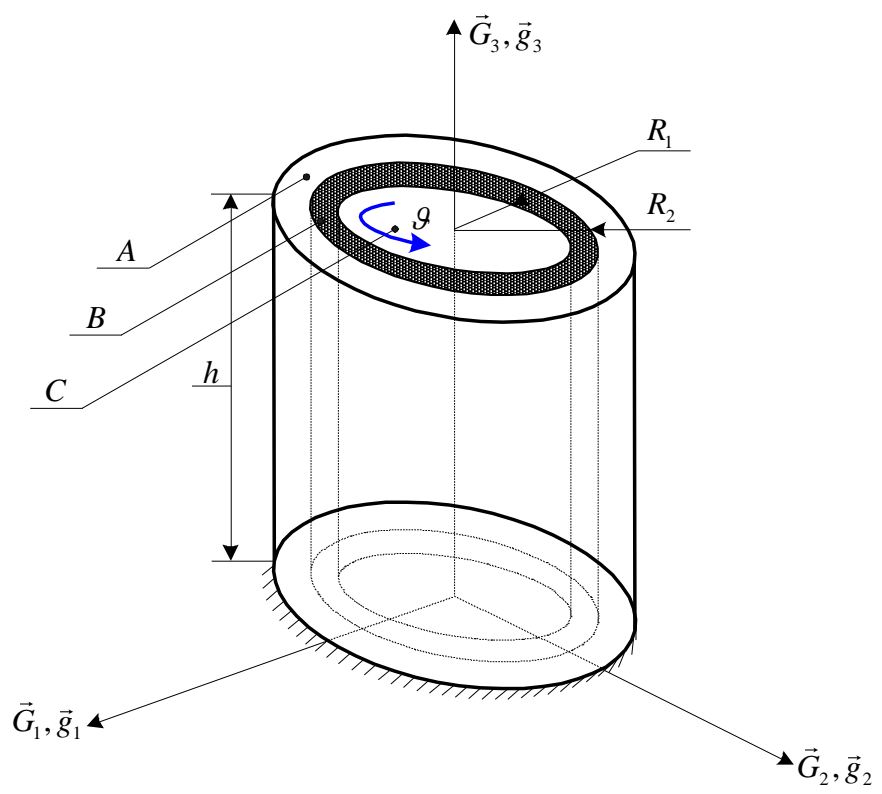

Figure 2. Mechanical modell of a silent block

The external body (A) is fixed and the internal one is imposed by a given rotation.

Further assumptions:

Planes perpendicular to the symmetric axis will be planes after the deformation.

The magnitude of the displacement is linear function of the measured distance from the axes of symmetry.

Furthermore, we are assuming that there aren't heat sources in the rubber and the temperature field shows homogeneous distribution, $h_{0}=0, q_{0}=0$.

We used the next material properties: $\mu=4,225 \cdot 10^{5}\left[\frac{\mathrm{N}}{\mathrm{m}^{2}}\right], \mathrm{c}=1580\left[\frac{\mathrm{Nm}}{\mathrm{kgK}}\right]$. Rotating the body $\mathrm{C}$ by angle $\vartheta$, we have assigned the displacement of a point in body $\mathrm{B}$ on an optional radius, Fig. 3 and we have determined the relationship 
between the reference and current configuration. Function $\vec{\chi}(\vec{R}, t)$ describing strain and characterizing the connection between the reference and current configuration has the following form on an optional radius:

$$
\begin{aligned}
& x=(\cos \varphi) X+(\sin \varphi) Y, \\
& y=(-\sin \varphi) X+(\cos \varphi) Y, \\
& z=Z
\end{aligned}, \vec{r}(t)=\vec{\chi}(\vec{R}, t),
$$

where $\varphi=\vartheta \frac{R_{2}-R}{R_{2}-R_{1}}, R=\sqrt{x^{2}+y^{2}}$.

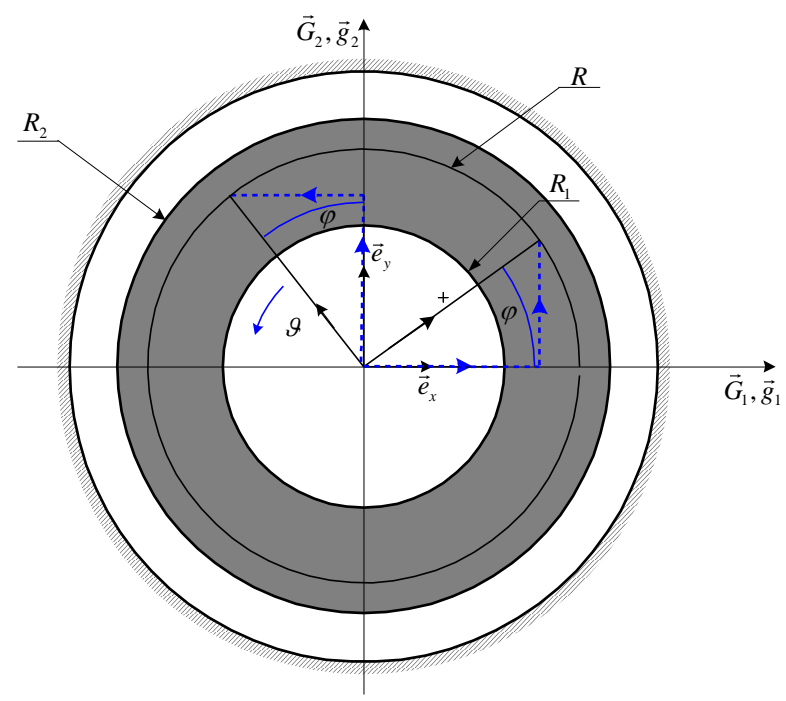

Figure 3. Plan view of the mechanical model of the silent block

The deformation gradient is obtained by the derivation of the function $\vec{\chi}(\vec{R}, t)$. Using this formula the problem of continuummechanics is regarded to be solved. Using of the deformation gradient we can derive the other quantities. We are assuming that the volume is constant so the deformation gradient can be expressed by the following way:

$$
\underline{\underline{F}}=\vec{\chi}(\vec{R}) \circ \nabla_{0},
$$


Applying the Eq. (20) the Cauchy stress tensor is:

$$
\underline{\underline{\sigma}}=\mu J^{-\frac{5}{3}}\left(\underline{\underline{b}}-\frac{1}{3} b_{I} \underline{\underline{I}}\right)+p \underline{\underline{I}},
$$

where $\mu$ is the shear modulus, $\underline{\underline{b}}=\underline{\underline{F}} \cdot \underline{\underline{F}}{ }^{T}$ is the left Cauchy-Green strain tensor, $b_{I}$ is the first scalar invariant of the left Cauchy-Green strain tensor, $J=\operatorname{det}(\underline{\underline{F}})=1$, $I$ is the identity tensor, $p$ is the pressure, which is the consequence of the constant volume, it can be determined by the boundary conditions, in this case by the condition $\sigma_{z}=0$.

After the substitution of the pressure $p$ the Cauchy-stress tensor is obtained in the next formulation:

$$
\underline{\underline{\sigma}}=\left[\begin{array}{ccc}
\sigma_{R} & \tau_{R \varphi} & 0 \\
\tau_{\varphi R} & \sigma_{\varphi} & 0 \\
0 & 0 & 0
\end{array}\right],
$$

The calculation of the stress values is determined by program wxMaxima, and using of Eq. (20).

The stress $\sigma_{R}$ is presented as the function of radius Fig. 4 and is compared with the simulation of the Ansys Finite Element Program. Results of both methods are in agreement with regard to their magnitude. One curve illustrates the changing of the values of stresses on the different radius of intermediate body B.

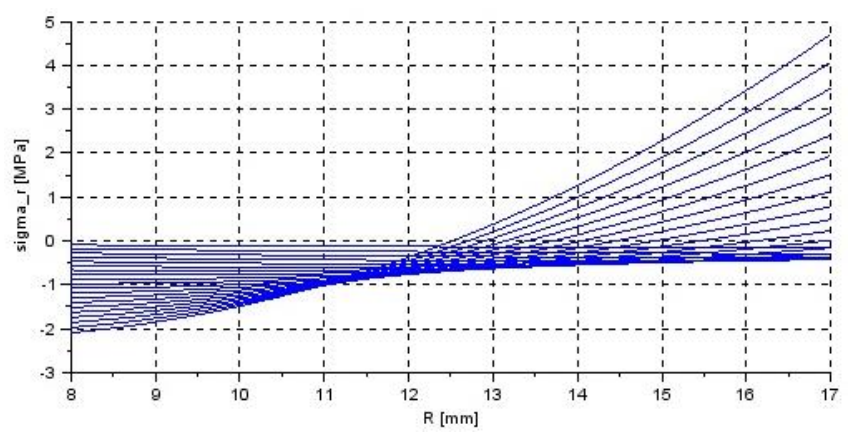

Figure 4. Representation of stress $\sigma_{R}$ as the function of radius 
The results of Fig. 4 illustrates very well the non-linear characterization of rubber's behaviour. Namely, decreasing or increasing of stress derives from the non-linear characterization.

Fig. 5 shows the result of the calculation by Ansys Finite Element Program.

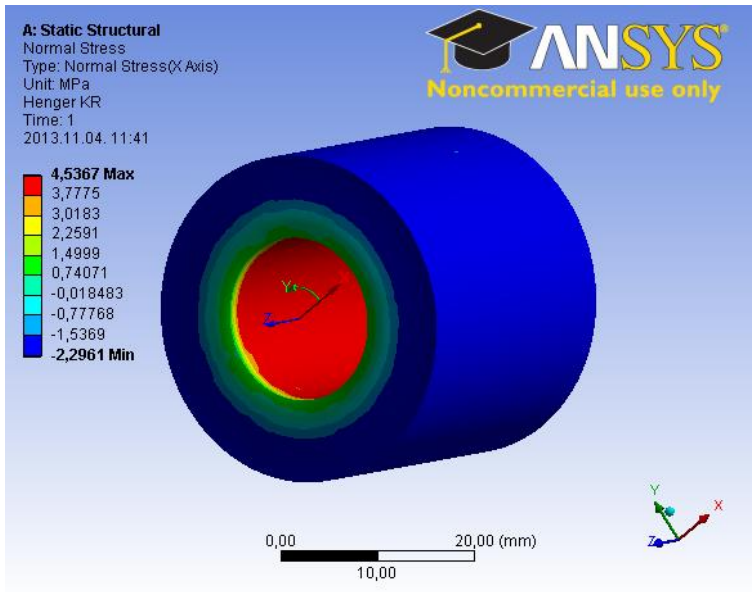

Figure 5. Representation of stress $\sigma_{R}$ as the function of radius

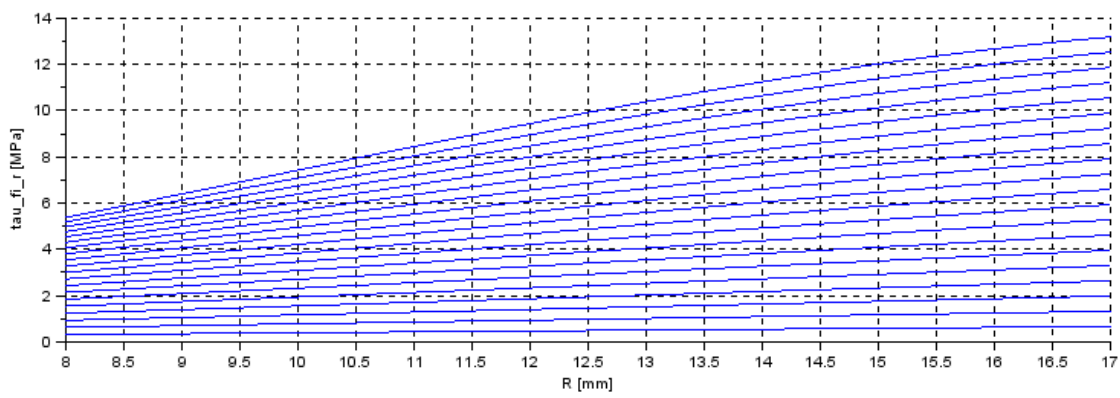

Figure 6. Representation of stress $\tau_{\varphi R}$ as the function of radius

The same values were calculated by in the cases of the stresses $\sigma_{\varphi}$ and $\tau_{\varphi R}$, like the same manner by using wxMaxima program and Scilab. The stress $\sigma_{\varphi}$ is presented as the function of radius Fig. 7. One curve illustrates the changing of the values of stresses on the different radius of intermediate body $\mathrm{B}$. 


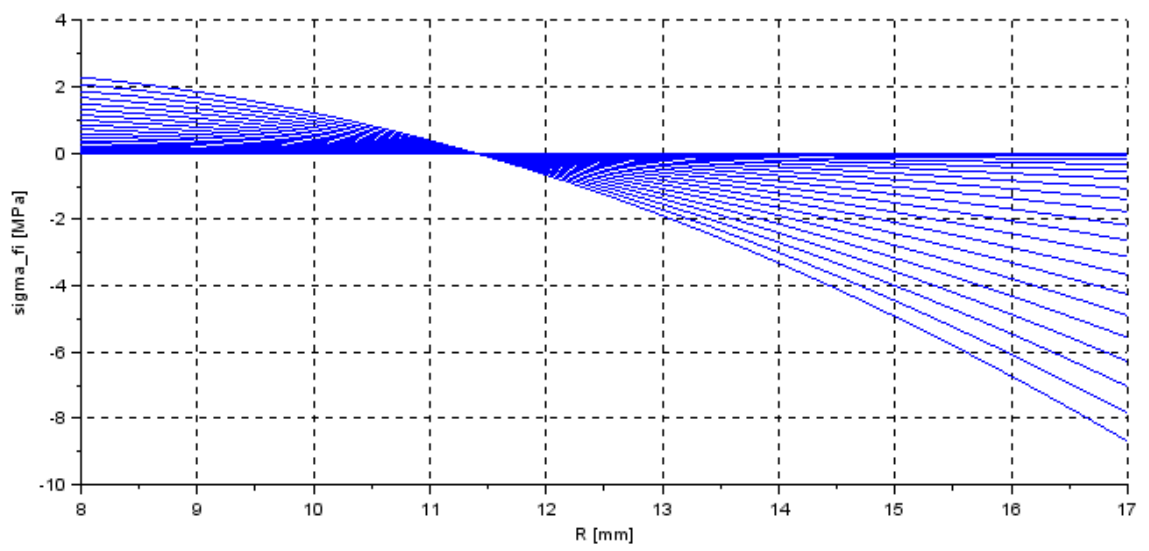

Figure 7. Representation of stress $\sigma_{\varphi}$ as the function of radius

The Fig.7 shows the results of calculation by Scilab. Hence, this figure illustrates the changing of the stress $\sigma_{\varphi}$ from radius $R_{1}$ to radius $R_{2}$ by the effect of the torsion of the internal body $\mathrm{C}$ from angle 0 to angle 10 .

Fig. 8 presents the temperature change by the effect of vibration frequency 1 Hertz.

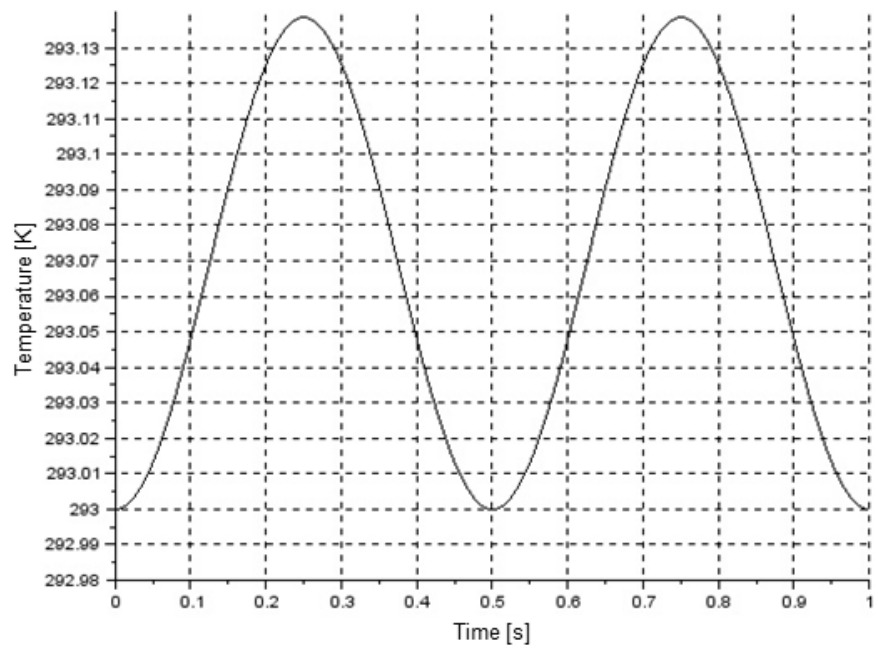

Figure 8. Temperature change as the function of time 
The calculation of temperature changes which is caused by the effect of vibration frequency 1 Hertz is obtained by the using of Eq.(16), i.e. $c \dot{T}=T \frac{\partial^{2} \psi}{\partial T \partial \underline{\underline{C}}} \cdot \cdot \underline{\underline{\dot{C}}}$. Substitution of the next relation to the Eq.(16), $\varphi=\vartheta \sin (\omega t) \frac{R_{2}-R}{R_{2}-R_{1}}$ the temperature changes can be calculated. Generation of calculations and the representation of the temperature changes as the function of time were determined by the SCILAB program.

\section{Summary}

We represented an algorythm which allows to calculate strain changes and temperature changes of the rubber part of the silent block under certain conditions. In the future we would like to develop a solving computer program in order to apply it as a thermodinamically consistent description.

\section{References}

[1] J. Bonet, R. D. Wood, Nonlinear Continuum Mechanics for Finite Element Analysis, Cambridge University Press, 1997.

[2] G. A. Holzapfel, Nonlinear Solid Mechanics, John Wiley\&Sons, Chichester, 2000 .

[3] R. W. Ogden, Large deformation isotropic elasticity: on the correlation of theory and experiment for incompressible rubberlike solids, Proceedings of the Royal Society A 326 (1567) (1972) pp. 565-584. doi: $10.1098 /$ rspa.1972.0026

[4] R. W. Ogden, Large deformation isotropic elasticity: on the correlation of theory and experiment for incompressible rubberlike solids, Proceedings of the Royal Society A 328 (1575) (1972) pp. 567-583. doi: 10.1098/rspa.1972.0096

[5] S. Reese, P. Wriggers, A material model for rubber-like polymers exhibiting plastic deformation: computational aspects and a comparison with experimental results, Computer Methods in Applied Mechanics and Engineering 148 (3-4) (1997), pp. 279-298. doi: 10.1016/S0045-7825(97)00034-0 
[6] M. Böl, S. Reese, Finite element modelling of rubber-like polymers based on chain statistics, International Journal of Solids and Structures 43 (1) (2006) pp. 2-26.

doi: 10.1016/j.ijsolstr.2005.06.086

[7] B. Pere, Solution of Coupled Thermomechanical Problems Using p-FEM, 8th European Solid Mechanics Conference (ESMC2012), Graz, Austria, 9-13 July 2012 (CD-ROM, 2 pages)

[8] G. A. Holzapfel, J. C. Simo: Entropy elasticity of isotropic rubber-like solids at finite strains, Computer Methods in Applied Mechanics and Engineering 132 (1-2) (1996), pp. 17-44.

doi: $10.1016 / 0045-7825(96) 01001-8$

[9] P. J. Flory, Statistical Mechanics of Chain Molecules, reprinted edition, Oxford University Press, New York, 1989.

[10] L.R.G. Treloar, The Physics of Rubber Elasticity, 3rd Edition, Oxford University Press, New York, 1975.

[11] S.C.H. Lu, K. S. Pister, Decomposition of deformation and representation of the free energy function for isotropic thermoelastic solids, International Journal of Solids and Structures 11 (7-8) (1975) pp. 927-934. doi: 10.1016/0020-7683(75)90015-3

[12] C. Miehe, Entropic thermoelasticity at finite strains. Aspects of formulation and numerical implementation, Computer Methods in Applied Mechanics and Engineering 120 (3-4) (1995) pp. 243-269. doi: 10.1016/0045-7825(94)00057-T 